\title{
MHC Class I Quality Control
}

\author{
Gustav Røder, Linda Geironson, Elna Follin, \\ Camilla Thuring and Kajsa Paulsson \\ Lund University \\ Sweden
}

\section{Introduction}

The immune system of higher organisms has the unique capability of mounting a specific and adaptive response against invading pathogens. Specific in the sense that immune cells recognize molecular features from foreign pathogens, and adaptive in the sense that upon reencounter of the foreign pathogen the immune system respond faster and more efficiently, which is the basic principle used in vaccination. In addition, the immune system has evolved the innate capability of recognizing common, slow mutating features on pathogens by using several different pattern recognition receptors. These defense mechanisms are important, but just like any other organisms pathogens also mutate to adapt to selection pressures thereby escaping immune surveillance. Likewise, the immune system of higher organisms is not a static system, but rather a dynamic ever-changing defense system continuously evolving over millions of years to constantly battle pathogens trying to overcome immune defense barriers and take over the host protein translation machinery. Furthermore, the immune system needs to be in balance with its host, but sometimes the line between functional immunity and auto-reactivity becomes hard to draw. Importantly, it is now a firmly established concept that the immune system eradicates host cells undergoing malignant transformation to prevent tumor formation. With these functions in mind, it is reasonable to claim the adaptive immune system as highly essential in the protection of pathogenic infections and malignant cells. The adaptive immune system contains a variety of T cells; two major groups are the $\mathrm{CD}^{+} \mathrm{T}$ cells that interact with MHC class II (MHC-II) cell surface receptors, and CD8 ${ }^{+} \mathrm{T}$ cells, also termed cytotoxic T cells (CTLs) that interact with MHC class I (MHC-I) cell surface receptors. The CTLs kill malignant host cells or host cells infected with foreign pathogens. This chapter focuses on the immunology concerning CTLs and MHC-I. Specifically, we focus on the multi-step, intracellular maturation of MHC-I.

CTLs continuously re-circulate throughout our body and secondary lymphoid organs to scan our cells for peptides presented on MHC-I. These peptides are derived from the interior of the cell, and presented on MHC-I outside the cell on its membrane surface. Each MHC-I receptor binds and presents one small endogenous or foreign peptide, which is derived from within the cell, (Roder, Geironson et al. 2008) and the entire ligand-receptor complex is termed a peptide-MHC-I complex (pMHC-I). Essentially, through their T cell receptor (TcR) the CTLs may recognize a particular pMHC-I on the cell surface of the presenting cell. The strength and outcome of this recognition depends on the identity of 
both the TcR and the pMHC-I, and occurs normally only when a foreign peptide is bound in the pMHC-I. This is the exact purpose of MHC-I defense system: use peptides to report any intracellular abnormalities such as infections or malignant states, and make the CTLs kill these cells. In the secondary lymphoid organs naïve CTLs encounter the pMHC-I complexes presented by professional APCs, cells with unique co-stimulatory capability able to prime naïve T cells. Upon recognition of a pMHC-I complex the CTLs become active dividing cells able to kill infected cells outside the lymphoid organs (Santana and Esquivel-Guadarrama 2006). An important characteristic of CTL activity is that it is pMHC-I restricted, giving that it only recognizes the peptide antigen in the context of a certain MHC-I molecule. Also, the pMHC-I is only recognized if it reaches and remains on the cell surface of the APC. If the pMHC-I disintegrates along the maturation and transport to, or while staying at, the cell surface it will never be available for CTL scrutiny. These criteria impose serious requirements to the intricate MHC-I maturation processes inside the cell as we shall describe in this chapter.

\section{The mature MHC-I binds only certain peptides in its binding groove}

Before we delve deep into the MHC-I maturation in the cell, we first introduce the reader to the final product - the mature pMHC-I complex and some of its important properties. MHC-I molecules are found on most nucleated cells in the body. The MHC-I in humans is called human leukocyte antigen class I (HLA-I) and is grouped into A, B and C based on the genetic locus. Because the protein products, the allomorphs, of the alleles occupying the HLA-A, -B and -C loci are all co-dominantly expressed and chromosomes exist in pairs, one from the paternal and one from the maternal side, usually six different allomorphs are expressed in each human. To date (2011), over 5,000 different alleles and over 3,000 different allomorphs have been identified and sequence information is freely available from the HLA Informatics Group (http://www.anthonynolan.com/HIG/data.html). As seen in figure 1 the final pMHC-I complex consists of a MHC-I heavy chain (HC) that is folded into three $\alpha$ domains; the $\alpha_{1}$ - and $\alpha_{2}$-domains are intertwined making up the membrane distal peptide binding groove, whereas the membrane proximal $\alpha_{3}$-domain has an Ig-like structure that supports binding of the MHC-I light chain, $\beta_{2}$-microglobulin $\left(\beta_{2} \mathrm{~m}\right)$. In addition, $\beta_{2} \mathrm{~m}$ stabilizes from below the anti-parallel $\beta$-sheets and $\alpha$-helices allowing these structural elements to create a large, but tight, peptide binding groove containing six peptide amino acid binding pockets.

These pockets are very important in determining the peptide binding specificity of any particular MHC-I allomorph. The MHC-I residues in each binding pocket determine, which peptide residues are compatible enough to bind in the pocket, and thus determine the requirement for particular residues at particular positions in the binding peptide. This is the MHC-I peptide binding specificity, and it turns out that only a few residue positions in the peptide are the major determinants of peptide binding specificity. Thus, only a small fraction of the entire peptide universe will be allowed to bind to any particular MHC-I allomorph, and it is this knowledge that is one important key to allow us to design peptidebased vaccines that are highly specific, cheap and easy to disseminate. In this respect, it is important to understand MHC-I maturation and the underlying criteria. 


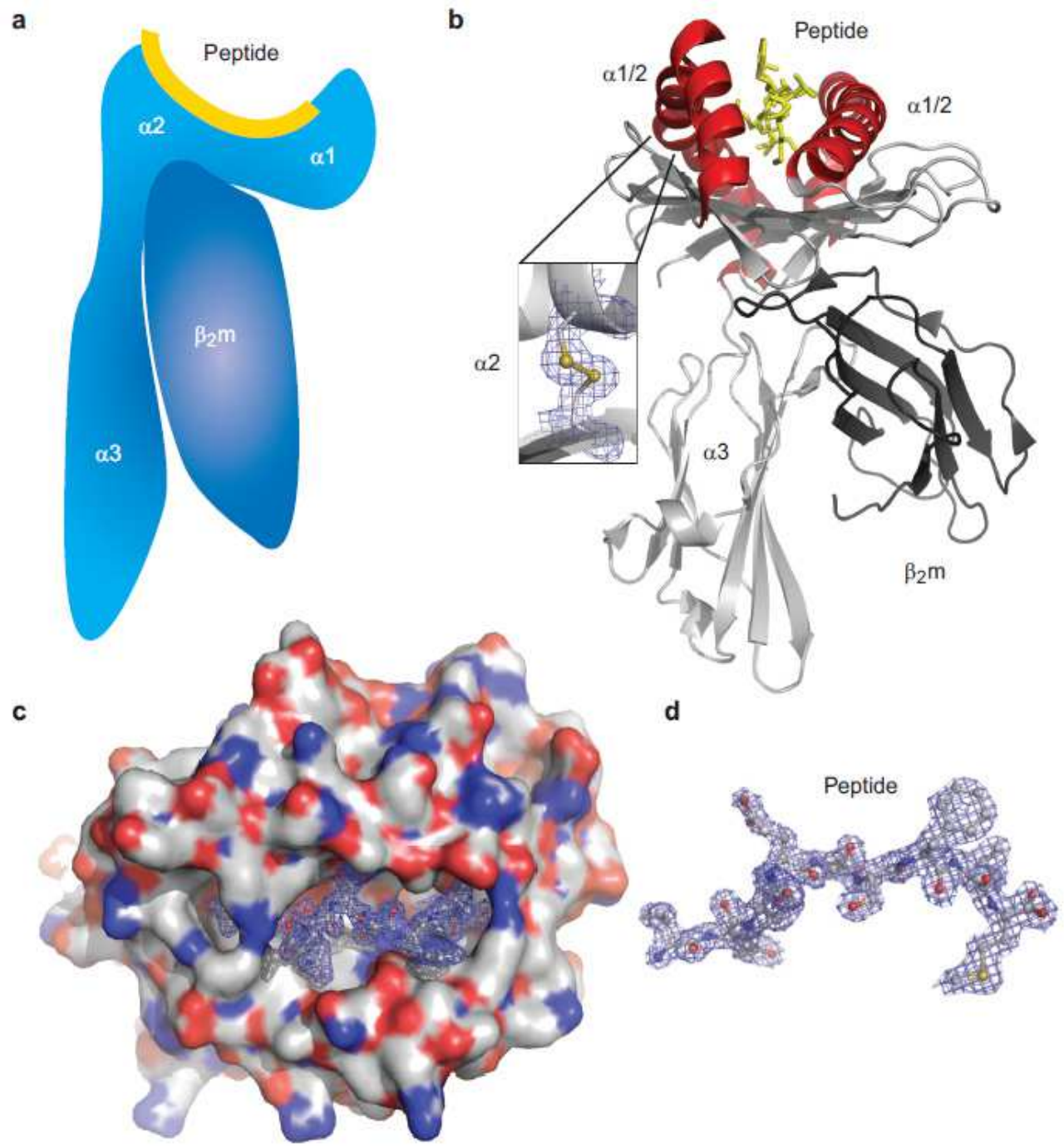

Fig. 1. The MHC-I protein structure allows short peptides to bind tightly in the peptide binding groove spanning the MHC-I $\alpha_{1} / \alpha_{2}$-domains. A) Schematic structure of MHC-I, which consists of a HC with $3 \alpha$-domains (light blue) and $\beta_{2} \mathrm{~m}$ (dark blue). In the peptide binding groove (at the top) the MHC-I has bound a peptide (yellow). B) The protein crystal structure of HLA-B*15:01. The HC is colored light grey and $\beta_{2} \mathrm{~m}$ is colored dark grey. The peptide binding groove is located at the top between two encapsulating $\alpha$-helices (red). The peptide (yellow) is seen from the C-terminal. The electron density of the Cys101-Cys164 disulfide bond in the peptide binding groove is shown in the inset. C) Top down view of the MHC-I peptide binding groove. D) The electron density of the peptide (LEKARGSTY). Shown from the $\alpha_{2}$-helix the peptide $\mathrm{N}$-terminal is at the left. 


\section{MHC-I matures and is loaded with peptide inside the cell}

The maturation of MHC-I and ultimate generation of pMHC-I complexes initially involves two different processes that later merge into one common maturation process. Firstly, the $\mathrm{HC}$ and $\beta_{2} \mathrm{~m}$ have to be produced separately and translocated into the endoplasmic reticulum (ER). At the same time, the peptide that will later bind to the MHC-I has to be produced and transported into the lumen of the ER. The entire MHC-I antigen processing machinery $(\mathrm{APM})$ process is illustrated in figure 2 showing the accessory proteins and chaperones involved in the stepwise processes ultimately resulting in a fully mature pMHCI complex that is exported to and situated on the cell surface.

Several APM components are involved in the MHC-I quality control cycle, and regulates the sequential maturation process of MHC-I (Paulsson and Wang 2004). The HC undergoes cotranslational glycosylation while being translocated into the ER lumen in a nascent unfolded state. Inside the ER the HC instantly interacts with $\mathrm{BiP}$, the Hsp90 protein, and soon also with calnexin, a general ER lectin chaperone. Calnexin binds to newly synthesized proteins with mono-glycosylated N-linked glycans, and in line with this also to early folding stages of the N-glycosylated HC (Ware, Vassilakos et al. 1995). This initial chaperoning of the HC prevents it from collapse and aggregation, and also sets the $\mathrm{HC}$ in a conformation that allows mature $\beta_{2} \mathrm{~m}$ to bind the HC forming the MHC-I heterodimer. Calnexin interacts with ERp57, a member of the protein disulfide isomerase (PDI) family, and is thought to involve in the correct formation of disulfide bonds within the HC (Oliver, Roderick et al. 1999). These intramolecular disulfide bonds strengthen the structural integrity and stability of the final pMHC-I complex. The newly formed MHC-I heterodimer is inherently unstable, but might be partly stabilized by binding a suboptimal peptide that later dissociates from the MHC-I. During these maturation steps calnexin dissociates from MHC-I and is immediately replaced by calreticulin, its soluble homologue.

At this stage the MHC-I might not be further matured due to the following reasons: 1) The MHC-I has acquired a peptide that induces final MHC-I maturation, 2) the particular MHC-I allomorph is inherently less prone to, or binds a peptide not inducing full maturation but inducing a structure not able to integrate into the peptide-loading complex (PLC), or 3), the MHC-I integration into the PLC is prohibited by viral interference (Roder, Geironson et al. 2008). However, for most HLA-I allomorphs the majority of MHC-I molecules are integrated into the PLC through binding to tapasin, where the late-stage maturation of the pMHC-I complex takes place. Tapasin has been ascribed many functions besides integrating MHC-I into the PLC. One of the most important functions, but also difficult to study, is to edit - and thereby optimize - the peptide loaded onto the MHC-I in the PLC with the purpose of generating stable pMHC-I complexes. In the PLC, which at least consists of the transporter associated with antigen processing (TAP), tapasin, calreticulin and ERp57, the MHC-I finally matures into a stable pMHC-I complex. ERp57 has proven important in the PLC, because it forms a disulfide conjugate with tapasin (Peaper, Wearsch et al. 2005). The tapasin-ERp57 disulfide conjugate has been suggested to significantly boost the efficiency, whereby tapasin edits the MHC-I bound peptide repertoire (Wearsch and Cresswell 2007).

So far, we have discussed the production and initial folding of the MHC-I. The generation and supply of peptides is equally important in the maturation of the final pMHC-I complex, and it all starts in the cytosol. Here, proteins are produced by the ribosome, but sometimes 
the production fails, resulting in defective ribosomal products (DRiPs). The DRiPs are targeted for degradation by the cytosolic proteasome, and it is from this cleavage process that peptides are generated. It has been proposed that a large fraction of the MHC-I binding peptides derive from DRiPs (Yewdell, Anton et al. 1996). Other cytosolic proteases, such as TPPII, may assist in trimming the proteasome processed peptides.
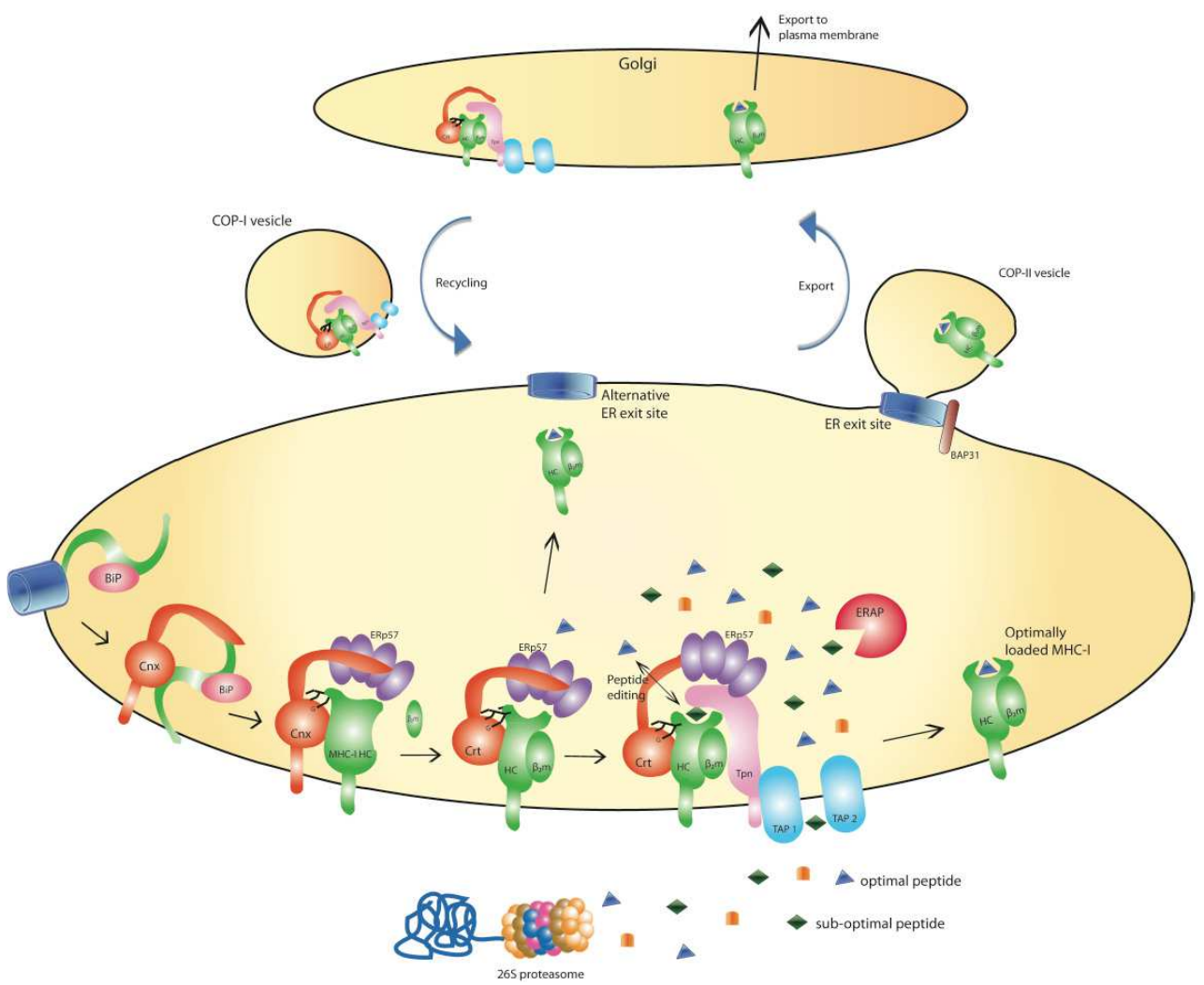

Fig. 2. An array of APM components present in the ER and the cytosol is involved in the sequential maturation and peptide loading of MHC-I. Early folding stages of MHC-I are promoted by BiP and then calnexin, which recruits ERp57. Subsequently, the MHC-I HC associates with $\beta_{2} \mathrm{~m}$, and calnexin is replaced by calreticulin. Most of the MHC-I molecules are recruited into the PLC, which consists of ERp57, calreticulin, tapasin and TAP, where the folding of the mature MHC-I conformation as well as peptide loading is facilitated. Peptides are generated in the cytosol by the proteasome and trimmed in the ER to MHC-I optimal lengths by ERAP1. MHC-I molecules loaded with optimal peptides become stable and can exit the ER and be transported via the Golgi network to the cell surface, possibly, between ER and Golgi, through transport in COP-II vesicles. Loading of an optimal peptide can also occur before recruitment into PLC. The stable pMHC-I complex can the exit the ER through alternative ER exit sites for transport to the cell surface. Sub-optimally loaded MHC-I molecules can escape the ER but are the recycled in COP-I vesicles recruited by tapasin, which recycles the MHC-I molecule back to the ER for optimal loading. 
An essential part of the PLC is the heterodimeric TAP, which is composed of the TAP1 and TAP2 subunits, both containing an N-terminal transmembrane domain and a C-terminal cytosolic nucleotide-binding domain. Situated in the ER membrane TAP actively translocates the cytosolic peptides into the ER in a multistep process, which begins with association of peptides, usually 8-16 amino acid long with TAP (van Endert, Tampe et al. 1994). Peptide binding to TAP is followed by an isomerization of the TAP complex that triggers an ATP-dependent peptide translocation across the ER membrane (Neefjes, Momburg et al. 1993). Many of the peptides that are transported by TAP into the ER lumen are still too long to bind firmly in the MHC-I peptide binding groove. These peptides are then further trimmed by the ER aminopeptidases ERAP1 and ERAP2 at the extended Nterminus to 8-10 amino acids, which is the appropriate length for association with MHC-I (York, Chang et al. 2002; Tanioka, Hattori et al. 2003).

We define an optimal MHC-I binding peptide as a peptide binding to MHC-I resulting in a stable pMHC-I complex. Thus, as a general rule, an optimal peptide has to bind firmly in the MHC-I peptide binding pockets. When a stable pMHC-I is formed, it is released from the PLC and transported to the cell surface. This underscores the importance of PLC-mediated pMHC-I quality control. pMHC-I complexes that consists only of suboptimal peptides are (per definition) unstable, and are not released from the PLC. This ensures the release of only stable pMHC-I complexes to the cell surface, which is vital for the subsequent CTL scrutiny. The pMHC-I transportation to the cell surface has been suggested to start with packaging of the pMHC-I complexes in COPII vesicles, which then migrate to the Golgi apparatus, and then further on to the cell surface (Paquet, Cohen-Doyle et al. 2004). The following sections discuss the MHC-I maturation, and peptide supply in much more detail.

\section{The MHC-I maturation is facilitated by multi-protein complexes in the ER}

In this section we go through the details of the HC production and folding in the ER involving APM components such as calnexin, calreticulin, ERp57 and tapasin. The chaperone BiP, known to interact cotranslationally with the nascent $\mathrm{HC}$ before other chaperones will not be further discussed. The following interactions of HC with each APM component are discussed in the maturation sequence as it is thought to occur.

\subsection{Calnexin and calreticulin shape the initial MHC-I heterodimer}

Both calnexin and its soluble homologue calreticulin are part of the general ER protein folding machinery, and bind to mono-glucosylated proteins. Despite overlapping substrate specificities, they also have distinct preferences, which can be explained by: 1) the positions and numbers of the glycosylation sites on the target protein (High, Lecomte et al. 2000), and 2) the membrane anchoring of calnexin versus the soluble calreticulin. The latter was shown to be a major determinant, since an experiment with soluble calnexin demonstrated that the glycoprotein association pattern for soluble calnexin resembles the pattern for calreticulin (Danilczyk, Cohen-Doyle et al. 2000). Similarly, calreticulin expressed with an ER membrane anchor binds to a set of glycoproteins resembling those proteins normally bound to calnexin (Wada, Imai et al. 1995). 


\subsubsection{Calnexin and calreticulin bind differently to MHC-I around the glycosylation site}

As alluded to in the previous section, the location of the glycosylation sequon in the MHC-I determines its binding to calnexin and calreticulin, which would then be reflected in the sequential maturation of MHC-I. Human MHC-I HCs have a single N-linked glycosylation site at Asn86, whereas mouse HC possess glycosylation sequons at additional sites (Maloy 1987). To support this notion, experiments have shown that introduction of a second glycosylation site in the human $\mathrm{HC}$ at the same position as in mouse $\mathrm{HC}$, resulted in a maturation process that more resembled the mouse HCs with respect to calnexin and calreticulin association (Zhang and Salter 1998). In the human, the HC glycosylation sequon is located close to the ER membrane during translocation into the ER, calnexin first gains access to this site. Calnexin induces $\beta_{2} \mathrm{~m}$ binding to the $\mathrm{HC}$, which brings the glycosylation sequon away from the membrane allowing the soluble calreticulin to replace calnexin (Solheim, Carreno et al. 1997).

In the $\beta_{2} \mathrm{~m}$ deficient human cell line Daudi, there is little or no association of MHC-I HC with calreticulin, suggesting that calreticulin only binds the MHC-I heterodimer, but not the single HC (Solheim, Harris et al. 1997; Tector, Zhang et al. 1997). In mice the situation is somewhat different, because calnexin not only binds to free $\mathrm{HC}$ but also to the $\mathrm{HC}$ heterodimer (Nossner and Parham 1995). Furthermore, it was shown that calnexin is associated with assembled mouse MHC-I even after peptide loading and dissociation from the PLC (Suh, Mitchell et al. 1996). Mouse HCs are able to bind to TAP also in the $\beta_{2} \mathrm{~m}$ deficient mouse cell line S3, and calnexin is present in this complex. Interestingly, also the fully folded human HLA-B*27 heterodimer has been shown to bind calnexin in human cells when using sensitive experimental systems, this is however outside the PLC (Carreno, Solheim et al. 1995). This shows, that the calnexin and calreticulin binding to MHC-I is not only species specific but also HC allomorph specific.

\subsubsection{Glycosylation and the calnexin/calreticulin cycle}

During the folding process, the MHC-I go through several rounds of dynamic binding, release and re-binding of calnexin/calreticulin in what is referred to the glycoprotein quality-control cycle (Helenius and Aebi 2004). The cycle is part of the general protein folding machinery in the ER, and is here illustrated in figure 3 in the context of MHC-I maturation. When bound calnexin or calreticulin is released from the HC, its glycosyl moiety becomes available for de-glucosylation by glucosidase II. Subsequently, the MHC-I folding status is evaluated by the soluble enzyme UDP-glucose glycosyl transferase (UGGT). If MHC-I is incorrectly folded the HC will be re-glycosylated and re-enter the glycoprotein quality control cycle (Helenius and Aebi 2004).

During translation and the BiP assisted folding the Asn86 of the MHC-I HC is N-glycosylated resulting in attachment of a $\mathrm{Glc}_{3} \mathrm{Man}_{9} \mathrm{GlcNAc}_{2}$ oligosaccharide moiety (Kornfeld and Kornfeld 1985). The two outer glucose residues are then removed by glycosidase I and II leaving the MHC-I HC mono-glucosylated ( $\mathrm{Gl}_{1} \mathrm{Man}_{9} \mathrm{GlCNAc}_{2}$ ) at this step in the maturation process (Elbein 1991). This enables subsequent interaction of HC with the lectin chaperones, calnexin and calreticulin (Hebert, Foellmer et al. 1995; Ware, Vassilakos et al. 1995).

Both calnexin and calreticulin bind mono-glucosylated substrates (Hammond, Braakman et al. 1994; Peterson, Ora et al. 1995). In addition they both interact with unfolded 


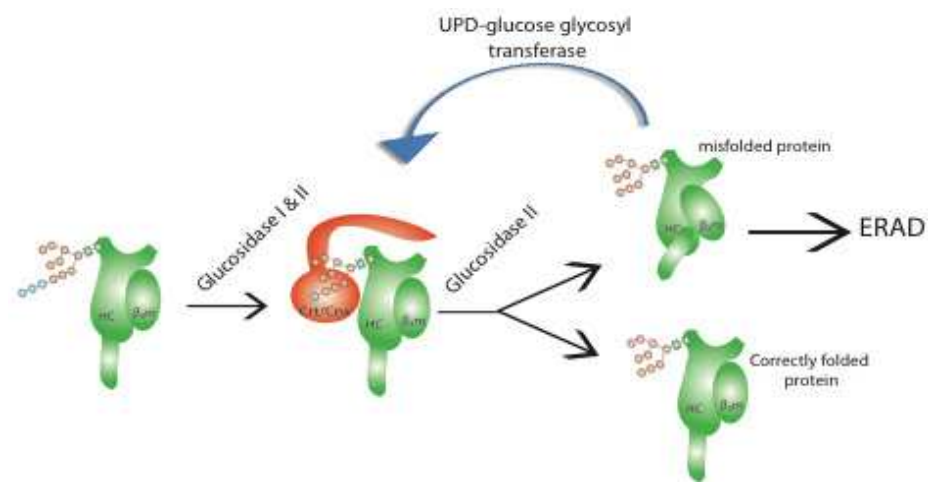

Fig. 3. The glycoprotein quality control cycle. Following the cotranslational glycosylation of MHC-I during translocation into the ER, glucosidase I and II remove the two outer glucoses immediately. When calnexin/calreticulin releases MHC-I the third glucose is removed and the folding status is evaluated by UDP-glucose glycosyl transferase. Incorrectly folded proteins will be re-glycosylated and re-enter the glycoprotein quality cycle whereas terminally misfolded proteins are degraded in the ER associated degradation (ERAD) pathway.

non-glucosylated proteins (Ihara, Cohen-Doyle et al. 1999; Saito, Ihara et al. 1999). As the newly synthesized MHC-I HC enters the ER via the translocon it is subjected to N-linked glycosylation of the sequon Asn-X-Ser/Thr (Bause, Muller et al. 1983). The glycosylation adds three glucose residues to the protein. The presence of a glycosylation site within the first 50 amino acids of a protein entering the ER through the translocon allows the interaction with calnexin and calreticulin to begin cotranslationally (Molinari and Helenius 2000). Inhibition of glycosylation results in many cases in misfolded, aggregated and dysfunctional proteins (Hickman, Kulczycki et al. 1977; Konig, Ashwell et al. 1988; Williams and Enns 1991). After trimming by glucosidase I and glucosidase II, mono-glucosylated HC is generated (Parodi 2000). The mono-glucosylated HC will then bind to calnexin for oxidative folding (Nossner and Parham 1995). Sequentially, the immature HC will create a heterodimer with the stabilising polypeptide $\beta_{2} \mathrm{~m}$. As this occurs, calnexin is replaced by calreticulin, which then recruits the immature heterodimer into the PLC where it can be loaded with high affinity peptides (Wearsch, Peaper et al. 2011).

It has been shown that the glycosylation of the MHC-I molecules in the ER is highly regulated. Nearly all monoglycosylated HC found in human cells are incorporated into the PLC and if deglycosylation is inhibited, their interaction with the PLC is significantly prolonged (Radcliffe, Diedrich et al. 2002; Wearsch, Peaper et al. 2011). A study using in vitro methods showed that glucosidase II could only trim free monyglycosylated HC and not those that were bound to the PLC (Wearsch, Peaper et al. 2011). This indicates that the glucose residues of the monoglycosylated MHC-I molecule become accessible to the glucosidase II first after its release from the PLC. After the last glucose residues has been removed, the MHC-I molecule can no longer associate with the PLC as its recruitment is dependent on the interaction with calreticulin which only binds to monoglycosylated proteins (Wearsch, Peaper et al. 2011). However, it has been proposed that if a deglycosylated MHC-I molecule is loaded with suboptimal peptide it can be rescued back into the PLC via the soluble enzyme UDP-glucose glycoprotein glucosyltransferase 1 
(UGT1) (Zhang, Wearsch et al. 2011). The UGT1 can selectively reglycosylate the suboptimally loaded/incorrectly folded MHC-I molecule, and after trimming by glucosidase I and glucosidase II the monoglycosylated MHC-I molecule can ones again enter the PLC, this what is called the calnexin/calreticulin cycle. After the MHC-I molecule has become correctly folded and loaded with an optimal peptide it will be deglycosylated and released from the calnexin/calreticulin cycle for transport from the ER to the cell surface for presentation (Radcliffe, Diedrich et al. 2002). The time spent in the calnexincalreticulin cycle can range from a few minutes to several hours, the more complex the folding of a protein, the more time it needs to spend. If a protein fails to reach mature conformation it is destined for ERAD (Klausner and Sitia 1990). Also for degradation tags the trimming of $\mathrm{N}$-linked glycans has been shown to be of importance and calnexin has been implicated in the sequestering of proteins tagged for degradation (Liu, Choudhury et al. 1997).

\subsubsection{Lack of calreticulin, but not calnexin, diminishes MHC-I quality control}

In normal human cells the PLC consist of calreticulin, tapasin, ERp57, TAP, maturing MHCI (Sadasivan, Lehner et al. 1996; Peaper, Wearsch et al. 2005). Ten years ago the hypothesis was that the abundant ERp57 found in the PLC was a consequence of its interaction with calreticulin or possibly calnexin (Oliver, Roderick et al. 1999). However, coimmunoprecipitation from K42 cells (a calreticulin deficient embryonic mouse fibroblast cell line) showed complexes of calnexin together with ERp57, tapasin and HC suggesting an alternative PLC constellation lacking calreticulin (Gao, Adhikari et al. 2002). Coimmunoprecipitation in lysates of K42 cells showed that ERp57 was associated with TAP, but that no calnexin could be detected in these precipitates, suggesting that neither calnexin nor calreticulin are essential for recruitment of ERp57 to the PLC (Suh, Mitchell et al. 1996; Gao, Adhikari et al. 2002). More recently it has been shown that ERp57 is directly disulfide conjugated to tapasin in the PLC (Peaper, Wearsch et al. 2005).

Calnexin deficient mice are viable and they show no discernible effects on other systems, including the immune system, possible because calreticulin effectively replaces the calnexin function even at the earlier MHC-I HC maturation stages. In contrast, calreticulin deficiency negatively impacts both trafficking and localization of MHC-I molecules. In the absence of calreticulin the MHC-I molecules are not retained in the ER, and the transport of immature MHC-I to the cell surface is accelerated. In K42 cells, many MHC-I molecules would be loaded with suboptimal peptides, due to lack of calreticulin in the MHC-I quality control, and the pMHC-I complexes would fall apart either before or on the cell surface (Gao, Adhikari et al. 2002). It was shown that the peptide-receptive MHC-I molecules were not found in the cis-Golgi or ER as they would be in wild type cells; instead they were found in endosomes and lysosomes. Under these circumstances the suboptimally loaded MHC-I molecules are transported to the cell surface, where the pMHC-I complexes almost instantly disintegrate, and are readily endocytosed ultimately leading to a significant reduction in MHC-I mediated antigen presentation (Howe, Garstka et al. 2009).

\subsection{ERp57 oxidizes the MHC-I heavy chain and supports tapasin function}

ERp57 is a multifunctional protein belonging to the thioredoxin family of proteins (Khanal and Nemere 2007). The protein structure is made up of four domains $a, b, a^{\prime}$ and $b^{\prime}$ (Ferrari 
and Soling 1999; Silvennoinen, Myllyharju et al. 2004). ERp57 is mainly, but not exclusively, an ER resident protein (Turano, Coppari et al. 2002) with a C-terminal Gln-Glu-Asp-Leu (QEDL) retention signal (Urade, Oda et al. 1997) and two thioredoxin domains with CGHC motifs shared with ERp72, protein disulfide isomerase (PDI) and other members of the thioredoxin family. Not only have many names been designated to this protein but several different functions of ERp57 have also been suggested including thiol-dependent oxidoreductase (Bourdi, Demady et al. 1995; Hirano, Shibasaki et al. 1995), cysteine protease (Urade and Kito 1992; Urade, Nasu et al. 1992), carnitine palmitoyl transferase (Wada, Imai et al. 1995), a hormone induced protein of the brain (Mobbs, Fink et al. 1990; Mobbs, Fink et al. 1990) and in combination with calnexin and/or calreticulin as a chaperone for $\mathrm{N}$ glycosylated proteins in the ER (Oliver, van der Wal et al. 1997).

In the ER, ERp57 has been shown to be associated with the PLC where it plays a critical role in the MHC-I maturation (Dong, Wearsch et al. 2009). It has also been shown to be involved in the quality control of other newly synthesized glycoproteins (Urade, Okudo et al. 2004; Khanal and Nemere 2007). In the PLC, ERp57 is covalently bound to tapasin via a disulfide bond between Cys95 of tapasin and Cys57 of ERp57, however the structural characterisation of the complex indicates that the heterodimer is also stabilised by non-covalent interactions between tapasin and the $a$ and $a^{\prime}$ domains of ERp57 (Dong, Wearsch et al. 2009). Calnexin and calreticulin, which both bind to ERp57 associate at the $b$ and $b^{\prime}$ domains of the protein (Russell, Ruddock et al. 2004; Kozlov, Maattanen et al. 2006). It has been suggested that the binding to calnexin and calreticulin is required for the recruitment of ERp57 into the PLC (Oliver, van der Wal et al. 1997; Oliver, Roderick et al. 1999). However, in more recent studies it has been proposed that ERp57 also on its own can recognize and bind some newly synthesized proteins such as MHC-I HC in the absence of calnexin and calreticulin (Zhang, Kozlov et al. 2009). An important function of ERp57 is its thiol-dependent reductase activity that catalyse the formation of protein disulide-bonds during the folding of glycoproteins in the ER (Tector and Salter 1995). However, in contrast, it has been shown that in the PLC and during the maturation of MHC-I, ERp57 has a more structural role by stabilising tapasin and the PLC (Garbi, Tanaka et al. 2006). The heterodimer of tapasin and ERp57 has also been shown to have a peptide editing function thereby playing a key role in the generation of mature and stable pMHC-I complexes that are to be presented on the cell surface (Wearsch and Cresswell 2007).

An ubiquitous deletion of the ERp57 gene in mouse results in death in utero. To study the effects of ERp57 deficiency this problem was circumvented by generating a tissue specific deletion in the B cell compartment that resulted in B cells lacking the expression of ERp57 (Garbi, Tanaka et al. 2006). The development and survival of the ERp57 deficient B cells were not affected nor was there any indication that the folding of glycoproteins such as the immunoglobulin, CD25, CD1d, CD19, CD23, CD72, CD40, CD80 and CD86 was reduced. This suggests that ERp57 is not essential for the basic B cell functions and glycoprotein folding in these mouse cells (Garbi, Tanaka et al. 2006). However, in another study the folding of specific glycoprotein substrates was shown to be reduced in the absence of ERp57 (Jessop, Chakravarthi et al. 2007). Even though the general glycoprotein folding was not shown to be affected in the ERp57 deficient B cells there was a significantly lower surface expression of MHC-I molecules $\left(\mathrm{H}-2 \mathrm{~K}^{\mathrm{b}}\right.$ showing a reduction of $50 \%$ whereas $\mathrm{H}-2 \mathrm{D}^{\mathrm{b}}$ was only slightly reduced) compared to non-B cells expressing wild type amounts of ERp57 
(Garbi, Tanaka et al. 2006). This further confirms the importance of ERp57 as a key player in generation of stable mature MHC-I molecules.

\subsection{Tapasin optimizes the MHC-I peptide repertoire by releasing only stable pMHC-I}

Tapasin (TAP associated glycoprotein) was discovered more than a decade ago and has during the years been assigned a central role in the loading aspects of the MHC-I quality control (Sadasivan, Lehner et al. 1996). In 1994, the description of the human lymphoblastoid B-cell line, termed LCL-721.220, marked the beginning of the discovery of tapasin. The LCL-721.220 did not have any HLA-A or HLA-B and only minor expression of HLA-C, and were devoid of full-length tapasin (Copeman, Bangia et al. 1998). The HLA-I cell surface expression of different HLA-I transfectants was monitored and found to be dependent on the particular HLA-I allomorph (Greenwood, Shimizu et al. 1994). For example, HLA-A*1 and HLA-B ${ }^{*} 8$ were reduced to $21 \%$ of the expression in a normal cell having tapasin, whereas HLA-A*2, HLA-A*3 and HLA-B*7 were reduced. The HLA-I cell surface expression was restored when LCL-721.220 was fused with either LCL-721.174 or Daudi; cell lines having tapasin. Thus, LCL-721.220 lacked one or several unknown mechanisms responsible for the maturation of MHC-I (Grandea, Androlewicz et al. 1995). At the same time, a $48 \mathrm{kDa}$ protein with a yet unknown function was shown to co-immunoprecipitate with TAP in a wild type cell line (Ortmann, Androlewicz et al. 1994). In 1996, this $48 \mathrm{kDa}$ protein was named tapasin (Sadasivan, Lehner et al. 1996), and it was shown by western blot to be absent in LCL-721.220 possibly explaining the defect in MHC-I maturation (Copeman, Bangia et al. 1998). Later, expression of tapasin in LCL-721.220 restored MHC-I cell surface expression and confirmed this hypothesis (Ortmann, Copeman et al. 1997). At that time, the true identity of tapasin was not known. The following year, the cDNA of the TAP-associated protein corresponding to tapasin was cloned, and it was found that tapasin is a 428 amino acid large, type-I transmembrane glycoprotein with a short cytoplasmic tail ( $\mathrm{Li}$, Sjogren et al. 1997; Ortmann, Copeman et al. 1997).

In 1997, it was proposed that tapasin is a member of the Ig superfamily containing an IgC1SET domain (Ortmann, Copeman et al. 1997; Herberg, Sgouros et al. 1998). Subsequently, it was suggested that amino acids 284 - 401 mostly originating from exon 5 has an Ig-like structure (Mayer and Klein 2001). This was further elaborated on using homology modeling suggesting that 287 - 401 has an Ig-like fold (Turnquist, Petersen et al. 2004). After many crystallization attempts, the first protein crystal structure was reported of tapasin in a stable disulfide conjugate with ERp57 (Dong, Wearsch et al. 2009). ERp57 has been proposed to stabilize tapasin when covalently associated through a disulfide bond (Peaper, Wearsch et al. 2005). The structure reveals that tapasin has two ER luminal domains. Domain- 1 is the Nterminal domain spanning residues 1-269, and domain-2 spans the remaining residues 270381 in the luminal part of tapasin.

To this date, it has not been possible to predict the structure of tapasin based on the amino acid sequence. This could be attributed to the fact that tapasin has a unique amino acid sequence especially within the now recognized domain-1. Related to the unique amino acid sequence the structure of tapasin domain-1 also has a unique 3-dimensional fold compared to other proteins. This domain consists mainly of anti-parallel beta-sheets. Interestingly, there are several ways to describe the structure of this domain. The most obvious regions 
within this domain are two beta-barrel-like structures that are tightly bound to each other. The stretch spanning the residues 77-102 contains a small alpha-helix (residues 83-90), but does not otherwise contain any secondary structure elements. The stretch contains the cysteine at position 95 and might serve as an extension from tapasin towards ERp57.

\subsubsection{Tapasin only integrates $\beta_{2} \mathrm{~m}$-associated MHC-I HC into the PLC}

Tapasin directly interacts with at least three components inside the PLC; it bridges MHC-I and TAP, and is simultaneously covalently bound to ERp57 thereby serving as an important structural component in the PLC. Tapasin does only interact with $\beta_{2} \mathrm{~m}$-associated MHC-I HC supported by observations showing that the MHC-I HC cannot be coimmunoprecipitated together with tapasin in Daudi cells, a $\beta_{2} \mathrm{~m}$ negative cell line (Paulsson, Wang et al. 2001). Furthermore, the PLC is not assembled in Daudi cells, strongly suggesting that the MHC-I maturation takes place in successive stages inside the ER, and that $\beta_{2} \mathrm{~m}$ is required for interaction with tapasin and integration into the PLC (Lewis and Elliott 1998). A contrasting study reported that tapasin directly interacts with MHC-I in the absence of $\beta_{2} \mathrm{~m}$ (Rizvi and Raghavan 2006). In the absence of other cellular components it was shown that recombinant tapasin directly interacts with MHC-I HC alone or the MHC-I heterodimer. The study also showed that sufficient $\beta_{2}$ m would effectively disrupt the tapasin-MHC-I interaction. In this regard, it may be speculated that in this in vitro system sufficient amounts of $\beta_{2} \mathrm{~m}$ may stabilize MHC-I to such an extent, that it is no longer unstable and is therefore not able to interact with tapasin.

\subsubsection{In the PLC, tapasin keeps MHC-I peptide-receptive}

Exactly how MHC-I gets loaded with (the right) peptide in the ER is controversial. MHC-I incorporation into the PLC is mediated by tapasin structurally bridging MHC-I and TAP (Li, Sjogren et al. 1997; Ortmann, Copeman et al. 1997). In addition to serving as a structural component in the PLC, tapasin has also been suggested to facilitate peptide loading, edit the MHC-I peptide cargo, retain and recycle sub-optimally loaded pMHC-I complexes (Grandea, Lehner et al. 1997; Paulsson, Kleijmeer et al. 2002; Zarling, Luckey et al. 2003; Paulsson, Jevon et al. 2006). Here, we will describe what is currently known about the role of tapasin in MHC-I quality control within the PLC.

To describe the chaperone and peptide-editor function of tapasin it is necessary to accurately define these terms. A chaperone is a protein that assists the folding of a target protein. Regarding tapasin, the only target protein identified is MHC-I, and the chaperone function of tapasin is to facilitate the folding of MHC-I. During MHC-I maturation, tapasin has been found to facilitate the assembly of MHC-I in the ER ultimately resulting in increased cell surface expression (Lauvau, Gubler et al. 1999; Zarling, Luckey et al. 2003; Everett and Edidin 2007). To support this notion, a study using recombinant tapasin and HLA-I showed that tapasin facilitates folding of HLA-I in the absence of other PLC proteins (Chen and Bouvier 2007). We have recently shown that tapasin has chaperone function, and that at least part of this chaperone function is located within the first 87 amino acids of tapasin $\left(\mathrm{Tpn}_{1-87}\right)$ (Roder, Geironson et al. 2009; Roder, Geironson et al. 2011). A cell-free assay was developed to study the $\mathrm{Tpn}_{1-87}$ chaperone function on folding HLA-I under controllable conditions. We observed that $\mathrm{Tpn}_{1-87}$ facilitated the folding of HLA-I, and the 
extend of the folding could be directly, but inversely, correlated with the intrinsic stability of the pMHC-I complex. Based on these observations a model for the tapasin chaperone function can be constructed in which, tapasin chaperones the MHC-I heterodimer by keeping it in a peptide-receptive state. A suboptimal peptide that only confers a lowstability pMHC-I complex will either not release bound tapasin (and thus be retained in the ER), or the pMHC-I will quickly disintegrate, and tapasin will instantly re-associate to the MHC-I heterodimer. Only optimal peptides will confer a highly stable pMHC-I complex and cause the release of tapasin.

Regarding the aforementioned peptide-editor function, it is here defined as tapasin catalyzing the exchange of peptides on MHC-I. More specifically, tapasin directly interacts with the MHC-I thereby keeping it in a peptide-receptive conformational state. Only when an optimal peptide is loaded onto the MHC-I will tapasin release the MHC-I as shown in a study using recombinant proteins (Rizvi and Raghavan 2006). Tapasin and ERp57 are disulfide linked in the PLC, and it was shown this tapasin-ERp57 disulfide conjugate catalyzes MHC-I peptide-exchange (Peaper, Wearsch et al. 2005). Interestingly, both tapasin chaperone and peptide-editor function can be thought of as consequences of the same underlying tapasin mechanism, which is tapasin assisted stabilization of MHC-I. Tapasin arrests the MHC-I in a conformation preventing the MHC-I from aggregation and degradation, the chaperone function. The same conformation also keeps the MHC-I in a peptide-receptive state, allowing the association and dissociation of peptides. Only an optimal peptide binding to the MHC-I is able to change the MHC-I conformation causing the release of MHC-I from tapasin. Taken together, chaperone and peptide-editor functions of tapasin could be considered the mere consequences of its interaction with the MHC-I.

One consequence of keeping the MHC-I in a peptide-receptive conformation is giving an increasing diversity of peptides a chance to bind to the MHC-I. Of course, in the ER most of these peptides will be of suboptimal nature. In this line of reason, tapasin is expected to alter the pool of peptides presented by MHC-I. This was observed in one study that reported a reduced number of peptides presented on HLA-A*02:01 in the absence of tapasin (Barber, Howarth et al. 2001). This observation supports the theory presented here, because in the absence of tapasin suboptimal peptides will out-compete the optimal peptide for binding to HLA-A*02:01, and the suboptimal pMHC-I complexes will still be transported to the cell surface, because tapasin is not present to retain them in the ER.

\subsubsection{Tapasin mediates recycling of unstable pMHC-I back to the ER}

Assurance of mature and highly stable pMHC-I complexes on the cell surface in wild-type cells has generally been attributed to ER retention of immature MHC-I. The established theory arises from different experimental systems showing that tapasin retains MHC-I molecules in the ER until loaded with optimal peptides, i.e. peptides binding to MHC-I and resulting in stable pMHC-I complexes (Schoenhals, Krishna et al. 1999; Grandea, Golovina et al. 2000).

In addition, a recycling mechanism of MHC-I from late secretory compartments back to the ER has been suggested to exist based on evidence from several studies (Hsu, Yuan et al. 1991; Bresnahan, Barber et al. 1997; Park, Lee et al. 2001; Paulsson, Kleijmeer et al. 2002). Shown in figure 2 are COP-I coated vesicles that recognize and bind to C-terminal KKXX-motifs in membrane proteins thereby functioning as ER retrieval signals for proteins (Cosson and 
Letourneur 1994). The findings that tapasin contains a C-terminal KKXX-motif and has been shown to have prolonged association with sub-optimally loaded pMHC-I complexes led to the investigation of tapasin involvement in COP-I transport (Paulsson, Kleijmeer et al. 2002). Tapasin was demonstrated to bind to COP-I via its KKXX-motif (Paulsson, Jevon et al. 2006). In cells expressing tapasin with the KKXX-motif mutated to AAXX, neither tapasin nor MHC-I were detected in association with COP-I, indicating a direct role for the tapasin KKXX-motif in mediating the MHC-I transport by COP-I coated vesicles. In the same cells, cell surface expression of MHC-I molecules was significantly increased, but MHC-I degradation was also increased suggesting escape to the cell surface of immature MHC-I. Thus, immature pMHC-I complexes having accidentally escaped the ER before being loaded with an optimal peptide, can be returned to the PLC in the ER for another round of peptide cargo optimization before being exported to the cell surface as mature and stable pMHC-I complexes.

\subsubsection{The maturation of different MHC-I allomorphs depends differently on tapasin}

A frequently used concept in the tapasin literature is tapasin dependency associated with each MHC-I allomorph. There has been a lack of previous consensus of this concept, and the use of dependency has been used referring to several types of distinct observations. In its strict sense, the dependency is how the cell surface expression of MHC-I depends on the presence of tapasin inside the cell. Thus, cell surface expression of HLA-I allomorphs that are highly dependent on tapasin will then be dramatically reduced in the absence of tapasin and vice versa. In this sense, HLA-I antigen presentation is controlled by tapasin to an extent depending on the HLA-I allomorph. To further investigate this dependency, we recently quantified the ability of $\mathrm{Tpn}_{1-87}$ to chaperone different pMHC-I complexes, see figure 4 . HLA-B*44:02 has been shown to be almost completely dependent on tapasin, since HLA$B^{*} 44: 02$ steady-state surface expression level is reduced by more than $90 \%$ in the tapasin deficient LCL-721.220 human cell line compared to the transfected homologues (Peh, Burrows et al. 1998). The same study also showed that HLA-B*27:05 surface expression was only slightly dependent on tapasin, whereas HLA-B*08:01 in this and another study was shown to be intermediate dependent on tapasin (Zarling, Luckey et al. 2003).
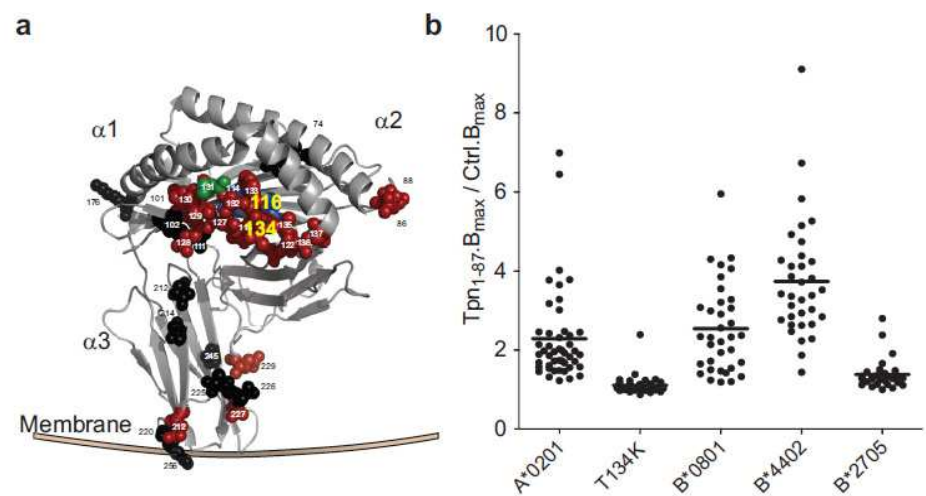

Fig. 4. MHC-I residues related to tapasin dependency (a), and tapasin chaperoning of different pMHC-I complexes (b). 
Regarding the specificity of the tapasin-MHC-I interaction, several reports have shown that entire regions and single residues on MHC-I and tapasin that are important in their interaction and function. The most well-studied single mutation was in HLA-A*02:01 where a threonine at position 134 was mutated to a lysine (T134K) (Lewis, Neisig et al. 1996; PeaceBrewer, Tussey et al. 1996). The HLA-A*02:01-T134K mutant allomorph is unable to interact with tapasin, and this mutation can thus be used as a negative control in experiments studying the tapasin- MHC -I interaction.

Notoriously, it is the amino acid sequence that makes MHC-I allomorph distinct. The amino acid at position 114 of HLA-B*44:02 and HLA-B*27:05 has been shown to be of crucial importance regarding tapasin dependence, the higher the acidity of this amino acid the higher the tapasin dependence (Park, Lee et al. 2003). HLA-B*27:05 and HLA-B*44:02 $(\mathrm{D} 114 \mathrm{H})$ both have histidine at position 114, and are both tapasin-independent, while HLA$\mathrm{B}^{*}$ 44:02 and HLA-B*35:01 have aspartic acid at residue 114 and are demonstrated to be dependent on tapasin (Peh, Burrows et al. 1998; Park, Lee et al. 2003). It is very interesting that a single residue (in certain HLA-I allomorphs) can completely change tapasin dependency, and it is especially interesting that residue 114 has this effect since it is not surface exposed, but buried deep down in the peptide binding groove close to the apex of the Fpocket. The tapasin interaction site on MHC-I is thought to be the surface exposed loop where the much discussed residue 134 is situated, and thus residue 114 is distal to the proposed tapasin interaction site, but is even though a strong determinant of tapasin dependence.

\subsubsection{Tapasin stabilizes TAP and increases peptide transport efficiency}

Tapasin binds directly to MHC-I, and form together with TAP the core of the PLC. The TAPtapasin complex interacts with MHC-I, calreticulin and ERp57 to form a fully functional PLC capable of loading peptides into the peptide-receptive MHC-I binding groove (Garbi, Tan et al. 2000; Gao, Adhikari et al. 2002; Williams, Peh et al. 2002; Garbi, Tanaka et al. 2006), see figure 2. The precise binding site to TAP has not yet been mapped, but it has been suggested that the first N-terminal transmembrane helix of TAP binds to the transmembrane domain of tapasin (Koch, Guntrum et al. 2006), supported by the fact that soluble human tapasin variants are defective in TAP association, also resulting in MHC-I that does not bind to TAP (Sadasivan, Lehner et al. 1996). As we will go in more detail with in a later section, TAP transports cytosolic peptides into the ER lumen. TAP exists as a TAP1/2 heterodimer, which was shown to be stabilized by tapasin (Garbi, Tiwari et al. 2003). Furthermore, tapasin was shown to increase the peptide transport throughput by TAP (Li, Paulsson et al. 2000). Thus, in addition to localizing MHC-I close to the source of peptide transportation, tapasin also structurally bridges the PLC together while at the same time interacts with and keeps the MHC-I in a peptide-receptive conformation.

\section{The MHC-I binding peptide has been processed by many proteases}

\subsection{The proteasome generates long peptides from degradable proteins}

Large multicatalytic protease complexes named proteasomes are located in the cytosol and degrade proteins as part of normal cellular turnover. The proteasome is essential for MHC-I antigen presentation, and inhibitors of the proteasome reduce the generation of peptides presented on MHC-I molecules (Rock, Gramm et al. 1994). Defective ribosomal products 
(DRiPs) are polypeptides that never reach a functional state owing to errors in translation or folding. They are rapidly ubiquitylated during translation and have been shown to be a major source of peptides presented by MHC-I (Reits, Vos et al. 2000; Schubert, Anton et al. 2000). The use of these newly translated proteins, facilitates rapid recruitment of effector cells to virally infected cells, resulting in a more efficient withdrawal of the infection.

The proteasome contains IFN- $\gamma$-inducible subunits. Under conditions of IFN- $\gamma$ induction the $\beta 1, \beta 2$ and $\beta 5$ subunits are replaced by immunosubunits, low-molecular-mass polypeptide 2 (LMP2), multi-catalytic endopeptidase complex-like 1 (MECL-1) and LMP7 respectively, resulting in assembly of new proteasomes called immunoproteasome (Loukissa, Cardozo et al. 2000; Jamaluddin, Wang et al. 2001). Immunoproteasomes are concentrated around the ER, whereas constitutive proteasomes are distributed evenly in the cytoplasm and in the nucleus (Brooks, Murray et al. 2000). Immunoproteasomes, compared to constitutive proteasomes, have an increased capacity to cleave peptides after hydrophobic and basic residues but reduced capacity to cleave after acidic amino acids (Gaczynska, Rock et al. 1993; Gaczynska, Rock et al. 1994). Furthermore, immunoproteasomes generate peptides with an extended Nterminal sequence that will facilitate transport into the ER (Cascio, Hilton et al. 2001; Knuehl, Spee et al. 2001). Even though immunoproteasomes favour the production of peptides presented by MHC-I molecules, they are not an absolute requirement (Arnold, Driscoll et al. 1992; Yewdell, Lapham et al. 1994), and some antigenic peptides are even efficiently produced by the constitutive proteasomes only (Chapiro, Claverol et al. 2006).

\subsection{TAP transports cytosolic peptides into the ER and supports MHC-I quality control}

An essential part of the PLC is the heterodimeric TAP composed of the TAP1 and TAP2 subunits, both containing an N-terminal transmembrane domain and a C-terminal cytosolic nucleotide-binding domain. TAP1 and TAP2 have 10 and 9 transmembrane helices, respectively, where the $6 \mathrm{C}$-terminal helices from each subunit build together to form the so called $6+6 \mathrm{TM}$ core complex which has been shown to be essential and sufficient for ER targeting, assembly of the heterodimer, binding of peptide and peptide translocation (Koch, Guntrum et al. 2004). The translocation is a multistep process, beginning with association of peptides with TAP in an ATP-independent manner (Androlewicz, Anderson et al. 1993; van Endert, Tampe et al. 1994; Neumann and Tampe 1999). Peptides with a length of 8-16 amino acids are preferentially bound to TAP (van Endert, Tampe et al. 1994). Peptides with 8-12 amino acids are transported most efficiently, although peptides longer than 40 amino acids are also transported, albeit with a lower efficiency (Androlewicz, Anderson et al. 1993; Koopmann, Post et al. 1996). The C-terminal amino acid and the first three N-terminal residues of the peptide have been shown to play a key role in TAP recognition (Scholz and Tampe 2005). Peptides with basic or hydrophobic amino acids at the C-terminus are particularly preferred by human TAP. Peptide binding to TAP is followed by a slow isomerization of the TAP complex that triggers an ATP-dependent peptide translocation across the ER membrane (Neefjes, Momburg et al. 1993; Shepherd, Schumacher et al. 1993; Scholz and Tampe 2005).

\subsection{Calreticulin and calnexin bind peptides - part of the peptide relay system}

As has been previously described both calnexin and calreticulin are multifunctional proteins. In addition to playing a key role in the maturation of MHC-I molecules via the 
calnexin/calreticulin cycle it has also been shown that they are able to bind peptides. Neefjes and colleagues have shown that both the chaperones calnexin and calreticulin bind peptides in vitro (Spee and Neefjes 1997; Spee, Subjeck et al. 1999). Calreticulin has also been shown to bind peptides in vivo (Nair, Wearsch et al. 1999). Many other heat shock proteins have also been demonstrated to bind peptide and a model of peptide transfer along a line of chaperones from the cytosol to the lumen of the ER - the peptide relay system - has been suggested to be responsible for directing peptides towards MHC-I presentation (Srivastava, Udono et al. 1994). The complexes formed when chaperones bind peptide have been suggested to bind to heat shock protein receptors on the surface of professional APCs, which leads to en internalization of the complex. The peptide from the complex is then presented by the MHC-I resulting in the elicitation of a CTL response. It has also been shown that chaperone-peptide complexes can stimulate the APCs to secrete certain cytokines (Srivastava, Menoret et al. 1998).

\subsection{ERAP aminopeptidases trim ER peptides to fit the MHC-I peptide binding groove}

After the peptides have been transported by TAP into the ER they may be further trimmed by aminopeptidases such as ER aminopeptidases 1 and 2 (ERAP1 and ERAP2) (Saric, Chang et al. 2002; Serwold, Gonzalez et al. 2002; Saveanu, Carroll et al. 2005). Although both are members of the M1 family of zinc metolloproteases (Rawlings, Barrett et al. 2010) ERAP1 and ERAP2 show striking differences in their substrate preferences. ERAP1 has a preference for large hydrophobic residues while ERAP2 prefers basic residues (Hattori, Kitatani et al. 2000; Tanioka, Hattori et al. 2003; Saveanu, Carroll et al. 2005). In contrast to most other aminopeptidases, that are more or less restricted to cleaving peptides shorter than four residues, ERAP1 shows a strong increase in cleaving activity towards peptides that are between 10-16 residues long (York, Chang et al. 2002). As ERAP1 and ERAP2 cleave substrates with different preferential for the N-terminal residues ERAP1 and ERAP2 have been suggested to work in concert for trimming of MHC-I binding peptides (Saveanu, Carroll et al. 2005).

The mechanism by with ERAP1 recognizes and cleaves peptides of a specific length has been debated. The crystal structure of ERAP1 (bound to bestatin) reveals a large cavity that would explain the preference for longer substrates compared to most other aminopeptidases (Nguyen, Chang et al. 2011). In this large cavity a catalytic site is found to which the Nterminal part of the peptide binds. It has been proposed that in close proximity to this catalytic site a regulatory site is found to which the C-terminal part of the peptide is bound, this "double binding" ensures a closed conformation of the ERAP1 molecule resulting in effective cleaving of the peptide. However, if the peptide is too short the C-terminal will not reach the regulatory site and the "double binding" will not be achieved resulting in an ineffective cleaving of the peptide (Nguyen, Chang et al. 2011).

The preference for longer immature antigenic peptide precursors supports the role of ERAP1 in the processing of antigenic peptides that are to be presented on MHC-I molecules, which preferentially bind peptides of eight to ten amino acid residues. It has been shown that over expression of ERAP1 enhances the presentation of antigenic peptides by MHC-I, confirming the importance of ERAP1 for MHC-I antigen presentation. 
In agreement with this is the finding from ERAP1 siRNA knock down studies that showed a reduction in antigenic peptides presented by the MHC-I molecules (York, Chang et al. 2002). In mice ERAP1 deficiency gives a significantly reduced MHC-I expression on the cell surface (Yan, Parekh et al. 2006). ERAP1 is IFN- $\gamma$ inducible as is many important APM components, putting ERAP1 in the same category (York, Chang et al. 2002).

\section{Perspectives}

Understanding the antigen processing and quality control of pMHC-I complexes is likely to significantly help us understand the criteria for why some MHC-I binding peptides are presented while others are not, and also why some peptides are able, and others are unable to function as CTL epitopes. The stability of pMHC-I complexes is likely to influence both positive and negative selection of responding CTLs implicating pMHC-I quality control as important in both autoimmune disease and as tumour antigens. Moreover, expression of many APM components is altered in many tumours and certain virus alter APM component activity and/or expression, and as a consequence change the MHC-I presented peptide pool both qualitatively and quantitatively. In conclusion further study of the APM and quality control of MHC-I will improve the identification of epitopes involved in both malignant neoplasms, viral diseases and in autoimmune diseases.

\section{References}

Androlewicz, M. J., K. S. Anderson, et al. (1993). "Evidence that transporters associated with antigen processing translocate a major histocompatibility complex class I-binding peptide into the endoplasmic reticulum in an ATP-dependent manner." Proceedings of the National Academy of Sciences of the United States of America 90(19): 9130-9134.

Arnold, D., J. Driscoll, et al. (1992). "Proteasome subunits encoded in the MHC are not generally required for the processing of peptides bound by MHC class I molecules." Nature 360(6400): 171-174.

Barber, L. D., M. Howarth, et al. (2001). "The quantity of naturally processed peptides stably bound by HLA-A*0201 is significantly reduced in the absence of tapasin." Tissue antigens 58(6): 363-368.

Bause, E., T. Muller, et al. (1983). "Synthesis and characterization of lipid-linked mannosyl oligosaccharides in Volvox carteri f. nagariensis." Archives of biochemistry and biophysics 220(1): 200-207.

Bourdi, M., D. Demady, et al. (1995). "cDNA cloning and baculovirus expression of the human liver endoplasmic reticulum P58: characterization as a protein disulfide isomerase isoform, but not as a protease or a carnitine acyltransferase." Archives of biochemistry and biophysics 323(2): 397-403.

Bresnahan, P. A., L. D. Barber, et al. (1997). "Localization of class I histocompatibility molecule assembly by subfractionation of the early secretory pathway." Human immunology 53(2): 129-139.

Brooks, P., R. Z. Murray, et al. (2000). "Association of immunoproteasomes with the endoplasmic reticulum." The Biochemical journal $352 \mathrm{Pt}$ 3: 611-615. 
Carreno, B. M., J. C. Solheim, et al. (1995). "TAP associates with a unique class I conformation, whereas calnexin associates with multiple class I forms in mouse and man." Journal of immunology 155(10): 4726-4733.

Cascio, P., C. Hilton, et al. (2001). "26S proteasomes and immunoproteasomes produce mainly N-extended versions of an antigenic peptide." The EMBO journal 20(10): 2357-2366.

Chapiro, J., S. Claverol, et al. (2006). "Destructive cleavage of antigenic peptides either by the immunoproteasome or by the standard proteasome results in differential antigen presentation." Journal of immunology 176(2): 1053-1061.

Chen, M. and M. Bouvier (2007). "Analysis of interactions in a tapasin/class I complex provides a mechanism for peptide selection." The EMBO journal 26(6): 1681-1690.

Copeman, J., N. Bangia, et al. (1998). "Elucidation of the genetic basis of the antigen presentation defects in the mutant cell line .220 reveals polymorphism and alternative splicing of the tapasin gene." European journal of immunology 28(11): 3783-3791.

Cosson, P. and F. Letourneur (1994). "Coatomer interaction with di-lysine endoplasmic reticulum retention motifs." Science 263(5153): 1629-1631.

Danilczyk, U. G., M. F. Cohen-Doyle, et al. (2000). "Functional relationship between calreticulin, calnexin, and the endoplasmic reticulum luminal domain of calnexin." The Journal of biological chemistry 275(17): 13089-13097.

Dong, G., P. A. Wearsch, et al. (2009). "Insights into MHC class I peptide loading from the structure of the tapasin-ERp57 thiol oxidoreductase heterodimer." Immunity 30(1): 21-32.

Elbein, A. D. (1991). "Glycosidase inhibitors: inhibitors of N-linked oligosaccharide processing." The FASEB journal : official publication of the Federation of American Societies for Experimental Biology 5(15): 3055-3063.

Everett, M. W. and M. Edidin (2007). "Tapasin increases efficiency of MHC I assembly in the endoplasmic reticulum but does not affect MHC I stability at the cell surface." Journal of immunology 179(11): 7646-7652.

Ferrari, D. M. and H. D. Soling (1999). "The protein disulphide-isomerase family: unravelling a string of folds." The Biochemical journal 339 ( Pt 1): 1-10.

Gaczynska, M., K. L. Rock, et al. (1993). "Gamma-interferon and expression of MHC genes regulate peptide hydrolysis by proteasomes." Nature 365(6443): 264-267.

Gaczynska, M., K. L. Rock, et al. (1994). "Peptidase activities of proteasomes are differentially regulated by the major histocompatibility complex-encoded genes for LMP2 and LMP7." Proceedings of the National Academy of Sciences of the United States of America 91(20): 9213-9217.

Gao, B., R. Adhikari, et al. (2002). "Assembly and antigen-presenting function of MHC class I molecules in cells lacking the ER chaperone calreticulin." Immunity 16(1): 99-109.

Garbi, N., P. Tan, et al. (2000). "Impaired immune responses and altered peptide repertoire in tapasin-deficient mice." Nature immunology 1(3): 234-238.

Garbi, N., S. Tanaka, et al. (2006). "Impaired assembly of the major histocompatibility complex class I peptide-loading complex in mice deficient in the oxidoreductase ERp57." Nature immunology 7(1): 93-102. 
Garbi, N., N. Tiwari, et al. (2003). "A major role for tapasin as a stabilizer of the TAP peptide transporter and consequences for MHC class I expression." European journal of immunology 33(1): 264-273.

Grandea, A. G., 3rd, M. J. Androlewicz, et al. (1995). "Dependence of peptide binding by MHC class I molecules on their interaction with TAP." Science 270(5233): 105-108.

Grandea, A. G., 3rd, T. N. Golovina, et al. (2000). "Impaired assembly yet normal trafficking of MHC class I molecules in Tapasin mutant mice." Immunity 13(2): 213-222.

Grandea, A. G., 3rd, P. J. Lehner, et al. (1997). "Regulation of MHC class I heterodimer stability and interaction with TAP by tapasin." Immunogenetics 46(6): 477-483.

Greenwood, R., Y. Shimizu, et al. (1994). "Novel allele-specific, post-translational reduction in HLA class I surface expression in a mutant human B cell line." Journal of immunology 153(12): 5525-5536.

Hammond, C., I. Braakman, et al. (1994). "Role of N-linked oligosaccharide recognition, glucose trimming, and calnexin in glycoprotein folding and quality control." Proceedings of the National Academy of Sciences of the United States of America 91(3): 913-917.

Hattori, A., K. Kitatani, et al. (2000). "Characterization of recombinant human adipocytederived leucine aminopeptidase expressed in Chinese hamster ovary cells." Journal of biochemistry 128(5): 755-762.

Hebert, D. N., B. Foellmer, et al. (1995). "Glucose trimming and reglucosylation determine glycoprotein association with calnexin in the endoplasmic reticulum." Cell 81(3): 425-433.

Helenius, A. and M. Aebi (2004). "Roles of N-linked glycans in the endoplasmic reticulum." Annual review of biochemistry 73: 1019-1049.

Herberg, J. A., J. Sgouros, et al. (1998). "Genomic analysis of the Tapasin gene, located close to the TAP loci in the MHC." European journal of immunology 28(2): 459-467.

Hickman, S., A. Kulczycki, Jr., et al. (1977). "Studies of the mechanism of tunicamycin in hibition of IgA and IgE secretion by plasma cells." The Journal of biological chemistry 252(12): 4402-4408.

High, S., F. J. Lecomte, et al. (2000). "Glycoprotein folding in the endoplasmic reticulum: a tale of three chaperones?" FEBS letters 476(1-2): 38-41.

Hirano, N., F. Shibasaki, et al. (1995). "Molecular cloning of the human glucose-regulated protein ERp57/GRP58, a thiol-dependent reductase. Identification of its secretory form and inducible expression by the oncogenic transformation." European journal of biochemistry / FEBS 234(1): 336-342.

Howe, C., M. Garstka, et al. (2009). "Calreticulin-dependent recycling in the early secretory pathway mediates optimal peptide loading of MHC class I molecules." The EMBO journal 28(23): 3730-3744.

Hsu, V. W., L. C. Yuan, et al. (1991). "A recycling pathway between the endoplasmic reticulum and the Golgi apparatus for retention of unassembled MHC class I molecules." Nature 352(6334): 441-444.

Ihara, Y., M. F. Cohen-Doyle, et al. (1999). "Calnexin discriminates between protein conformational states and functions as a molecular chaperone in vitro." Molecular cell 4(3): 331-341. 
Jamaluddin, M., S. Wang, et al. (2001). "IFN-beta mediates coordinate expression of antigenprocessing genes in RSV-infected pulmonary epithelial cells." American journal of physiology. Lung cellular and molecular physiology 280(2): L248-257.

Jessop, C. E., S. Chakravarthi, et al. (2007). "ERp57 is essential for efficient folding of glycoproteins sharing common structural domains." The EMBO journal 26(1): 28-40.

Khanal, R. C. and I. Nemere (2007). "The ERp57/GRp58/1,25D3-MARRS receptor: multiple functional roles in diverse cell systems." Current medicinal chemistry 14(10): 10871093.

Klausner, R. D. and R. Sitia (1990). "Protein degradation in the endoplasmic reticulum." Cell 62(4): 611-614.

Knuehl, C., P. Spee, et al. (2001). "The murine cytomegalovirus pp89 immunodominant H2Ld epitope is generated and translocated into the endoplasmic reticulum as an 11mer precursor peptide." Journal of immunology 167(3): 1515-1521.

Koch, J., R. Guntrum, et al. (2004). "Functional dissection of the transmembrane domains of the transporter associated with antigen processing (TAP)." The Journal of biological chemistry 279(11): 10142-10147.

Koch, J., R. Guntrum, et al. (2006). "The first N-terminal transmembrane helix of each subunit of the antigenic peptide transporter TAP is essential for independent tapasin binding." FEBS letters 580(17): 4091-4096.

Konig, R., G. Ashwell, et al. (1988). "Glycosylation of CD4. Tunicamycin inhibits surface expression." The Journal of biological chemistry 263(19): 9502-9507.

Koopmann, J. O., M. Post, et al. (1996). "Translocation of long peptides by transporters associated with antigen processing (TAP)." European journal of immunology 26(8): $1720-1728$.

Kornfeld, R. and S. Kornfeld (1985). "Assembly of asparagine-linked oligosaccharides." Annual review of biochemistry 54: 631-664.

Kozlov, G., P. Maattanen, et al. (2006). "Crystal structure of the bb' domains of the protein disulfide isomerase ERp57." Structure 14(8): 1331-1339.

Lauvau, G., B. Gubler, et al. (1999). "Tapasin enhances assembly of transporters associated with antigen processing-dependent and -independent peptides with HLA-A2 and HLA-B27 expressed in insect cells." The Journal of biological chemistry 274(44): 3134931358.

Lewis, J. W. and T. Elliott (1998). "Evidence for successive peptide binding and quality control stages during MHC class I assembly." Current biology : CB 8(12): 717-720.

Lewis, J. W., A. Neisig, et al. (1996). "Point mutations in the alpha 2 domain of HLA-A2.1 define a functionally relevant interaction with TAP." Current biology : CB 6(7): 873883.

Li, S., K. M. Paulsson, et al. (2000). "Tapasin is required for efficient peptide binding to transporter associated with antigen processing." The Journal of biological chemistry 275(3): 1581-1586.

Li, S., H. O. Sjogren, et al. (1997). "Cloning and functional characterization of a subunit of the transporter associated with antigen processing." Proceedings of the National Academy of Sciences of the United States of America 94(16): 8708-8713.

Liu, Y., P. Choudhury, et al. (1997). "Intracellular disposal of incompletely folded human alpha1-antitrypsin involves release from calnexin and post-translational trimming 
of asparagine-linked oligosaccharides." The Journal of biological chemistry 272(12): 7946-7951.

Loukissa, A., C. Cardozo, et al. (2000). "Control of LMP7 expression in human endothelial cells by cytokines regulating cellular and humoral immunity." Cytokine 12(9): 13261330.

Maloy, W. L. (1987). "Comparison of the primary structure of class I molecules." Immunologic research 6(1-2): 11-29.

Mayer, W. E. and J. Klein (2001). "Is tapasin a modified Mhc class I molecule?" Immunogenetics 53(9): 719-723.

Mobbs, C. V., G. Fink, et al. (1990). "HIP-70: a protein induced by estrogen in the brain and LH-RH in the pituitary." Science 247(4949 Pt 1): 1477-1479.

Mobbs, C. V., G. Fink, et al. (1990). "HIP-70: an isoform of phosphoinositol-specific phospholipase C-alpha." Science 249(4968): 566-567.

Molinari, M. and A. Helenius (2000). "Chaperone selection during glycoprotein translocation into the endoplasmic reticulum." Science 288(5464): 331-333.

Nair, S., P. A. Wearsch, et al. (1999). "Calreticulin displays in vivo peptide-binding activity and can elicit CTL responses against bound peptides." Journal of immunology 162(11): 6426-6432.

Neefjes, J. J., F. Momburg, et al. (1993). "Selective and ATP-dependent translocation of peptides by the MHC-encoded transporter." Science 261(5122): 769-771.

Neumann, L. and R. Tampe (1999). "Kinetic analysis of peptide binding to the TAP transport complex: evidence for structural rearrangements induced by substrate binding." Journal of molecular biology 294(5): 1203-1213.

Nguyen, T. T., S. C. Chang, et al. (2011). "Structural basis for antigenic peptide precursor processing by the endoplasmic reticulum aminopeptidase ERAP1." Nature structural \& molecular biology 18(5): 604-613.

Nossner, E. and P. Parham (1995). "Species-specific differences in chaperone interaction of human and mouse major histocompatibility complex class I molecules." The Journal of experimental medicine 181(1): 327-337.

Oliver, J. D., H. L. Roderick, et al. (1999). "ERp57 functions as a subunit of specific complexes formed with the ER lectins calreticulin and calnexin." Molecular biology of the cell 10(8): 2573-2582.

Oliver, J. D., F. J. van der Wal, et al. (1997). "Interaction of the thiol-dependent reductase ERp57 with nascent glycoproteins." Science 275(5296): 86-88.

Ortmann, B., M. J. Androlewicz, et al. (1994). "MHC class I/beta 2-microglobulin complexes associate with TAP transporters before peptide binding." Nature 368(6474): 864-867.

Ortmann, B., J. Copeman, et al. (1997). "A critical role for tapasin in the assembly and function of multimeric MHC class I-TAP complexes." Science 277(5330): 1306-1309.

Paquet, M. E., M. Cohen-Doyle, et al. (2004). "Bap29/31 influences the intracellular traffic of MHC class I molecules." Journal of immunology 172(12): 7548-7555.

Park, B., S. Lee, et al. (2003). "A single polymorphic residue within the peptide-binding cleft of MHC class I molecules determines spectrum of tapasin dependence." Journal of immunology 170(2): 961-968.

Park, B., S. Lee, et al. (2001). "The truncated cytoplasmic tail of HLA-G serves a qualitycontrol function in post-ER compartments." Immunity 15(2): 213-224. 
Parodi, A. J. (2000). "Role of N-oligosaccharide endoplasmic reticulum processing reactions in glycoprotein folding and degradation." The Biochemical journal $348 \mathrm{Pt}$ 1: 1-13.

Paulsson, K. M., M. Jevon, et al. (2006). "The double lysine motif of tapasin is a retrieval signal for retention of unstable MHC class I molecules in the endoplasmic reticulum." Journal of immunology 176(12): 7482-7488.

Paulsson, K. M., M. J. Kleijmeer, et al. (2002). "Association of tapasin and COPI provides a mechanism for the retrograde transport of major histocompatibility complex (MHC) class I molecules from the Golgi complex to the endoplasmic reticulum." The Journal of biological chemistry 277(21): 18266-18271.

Paulsson, K. M. and P. Wang (2004). "Quality control of MHC class I maturation." The FASEB journal : official publication of the Federation of American Societies for Experimental Biology 18(1): 31-38.

Paulsson, K. M., P. Wang, et al. (2001). "Distinct differences in association of MHC class I with endoplasmic reticulum proteins in wild-type, and beta 2-microglobulin- and TAP-deficient cell lines." International immunology 13(8): 1063-1073.

Peace-Brewer, A. L., L. G. Tussey, et al. (1996). "A point mutation in HLA-A*0201 results in failure to bind the TAP complex and to present virus-derived peptides to CTL." Immunity 4(5): 505-514.

Peaper, D. R., P. A. Wearsch, et al. (2005). "Tapasin and ERp57 form a stable disulfide-linked dimer within the MHC class I peptide-loading complex." The EMBO journal 24(20): 3613-3623.

Peh, C. A., S. R. Burrows, et al. (1998). "HLA-B27-restricted antigen presentation in the absence of tapasin reveals polymorphism in mechanisms of HLA class I peptide loading." Immunity 8(5): 531-542.

Peterson, J. R., A. Ora, et al. (1995). "Transient, lectin-like association of calreticulin with folding intermediates of cellular and viral glycoproteins." Molecular biology of the cell 6(9): 1173-1184.

Radcliffe, C. M., G. Diedrich, et al. (2002). "Identification of specific glycoforms of major histocompatibility complex class I heavy chains suggests that class I peptide loading is an adaptation of the quality control pathway involving calreticulin and ERp57." The Journal of biological chemistry 277(48): 46415-46423.

Rawlings, N. D., A. J. Barrett, et al. (2010). "MEROPS: the peptidase database." Nucleic acids research 38(Database issue): D227-233.

Reits, E. A., J. C. Vos, et al. (2000). "The major substrates for TAP in vivo are derived from newly synthesized proteins." Nature 404(6779): 774-778.

Rizvi, S. M. and M. Raghavan (2006). "Direct peptide-regulatable interactions between MHC class I molecules and tapasin." Proceedings of the National Academy of Sciences of the United States of America 103(48): 18220-18225.

Rock, K. L., C. Gramm, et al. (1994). "Inhibitors of the proteasome block the degradation of most cell proteins and the generation of peptides presented on MHC class I molecules." Cell 78(5): 761-771.

Roder, G., L. Geironson, et al. (2008). "Viral proteins interfering with antigen presentation target the major histocompatibility complex class I peptide-loading complex." Journal of virology 82(17): 8246-8252. 
Roder, G., L. Geironson, et al. (2009). "The outermost N-terminal region of tapasin facilitates folding of major histocompatibility complex class I." European journal of immunology 39(10): 2682-2694.

Roder, G., L. Geironson, et al. (2011). "Tapasin discriminates peptide-human leukocyte antigen- $A^{*} 02: 01$ complexes formed with natural ligands." The Journal of biological chemistry 286(23): 20547-20557.

Russell, S. J., L. W. Ruddock, et al. (2004). "The primary substrate binding site in the b' domain of ERp57 is adapted for endoplasmic reticulum lectin association." The Journal of biological chemistry 279(18): 18861-18869.

Sadasivan, B., P. J. Lehner, et al. (1996). "Roles for calreticulin and a novel glycoprotein, tapasin, in the interaction of MHC class I molecules with TAP." Immunity 5(2): 103114.

Saito, Y., Y. Ihara, et al. (1999). "Calreticulin functions in vitro as a molecular chaperone for both glycosylated and non-glycosylated proteins." The EMBO journal 18(23): 67186729.

Santana, M. A. and F. Esquivel-Guadarrama (2006). "Cell biology of T cell activation and differentiation." International review of cytology 250: 217-274.

Saric, T., S. C. Chang, et al. (2002). "An IFN-gamma-induced aminopeptidase in the ER, ERAP1, trims precursors to MHC class I-presented peptides." Nature immunology 3(12): 1169-1176.

Saveanu, L., O. Carroll, et al. (2005). "Concerted peptide trimming by human ERAP1 and ERAP2 aminopeptidase complexes in the endoplasmic reticulum." Nature immunology 6(7): 689-697.

Schoenhals, G. J., R. M. Krishna, et al. (1999). "Retention of empty MHC class I molecules by tapasin is essential to reconstitute antigen presentation in invertebrate cells." The EMBO journal 18(3): 743-753.

Scholz, C. and R. Tampe (2005). "The intracellular antigen transport machinery TAP in adaptive immunity and virus escape mechanisms." Journal of bioenergetics and biomembranes 37(6): 509-515.

Schubert, U., L. C. Anton, et al. (2000). "Rapid degradation of a large fraction of newly synthesized proteins by proteasomes." Nature 404(6779): 770-774.

Serwold, T., F. Gonzalez, et al. (2002). "ERAAP customizes peptides for MHC class I molecules in the endoplasmic reticulum." Nature 419(6906): 480-483.

Shepherd, J. C., T. N. Schumacher, et al. (1993). "TAP1-dependent peptide translocation in vitro is ATP dependent and peptide selective." Cell 74(3): 577-584.

Silvennoinen, L., J. Myllyharju, et al. (2004). "Identification and characterization of structural domains of human ERp57: association with calreticulin requires several domains." The Journal of biological chemistry 279(14): 13607-13615.

Solheim, J. C., B. M. Carreno, et al. (1997). "Are transporter associated with antigen processing (TAP) and tapasin class I MHC chaperones?" Journal of immunology 158(2): 541-543.

Solheim, J. C., M. R. Harris, et al. (1997). "Prominence of beta 2-microglobulin, class I heavy chain conformation, and tapasin in the interactions of class I heavy chain with calreticulin and the transporter associated with antigen processing." Journal of immunology 158(5): 2236-2241. 
Spee, P. and J. Neefjes (1997). "TAP-translocated peptides specifically bind proteins in the endoplasmic reticulum, including gp96, protein disulfide isomerase and calreticulin." European journal of immunology 27(9): 2441-2449.

Spee, P., J. Subjeck, et al. (1999). "Identification of novel peptide binding proteins in the endoplasmic reticulum: ERp72, calnexin, and grp170." Biochemistry 38(32): 1055910566.

Srivastava, P. K., A. Menoret, et al. (1998). "Heat shock proteins come of age: primitive functions acquire new roles in an adaptive world." Immunity 8(6): 657-665.

Srivastava, P. K., H. Udono, et al. (1994). "Heat shock proteins transfer peptides during antigen processing and CTL priming." Immunogenetics 39(2): 93-98.

Suh, W. K., E. K. Mitchell, et al. (1996). "MHC class I molecules form ternary complexes with calnexin and TAP and undergo peptide-regulated interaction with TAP via their extracellular domains." The Journal of experimental medicine 184(2): 337-348.

Tanioka, T., A. Hattori, et al. (2003). "Human leukocyte-derived arginine aminopeptidase. The third member of the oxytocinase subfamily of aminopeptidases." The Journal of biological chemistry 278(34): 32275-32283.

Tector, M. and R. D. Salter (1995). "Calnexin influences folding of human class I histocompatibility proteins but not their assembly with beta 2-microglobulin." The Journal of biological chemistry 270(33): 19638-19642.

Tector, M., Q. Zhang, et al. (1997). "Beta 2-microglobulin and calnexin can independently promote folding and disulfide bond formation in class I histocompatibility proteins." Molecular immunology 34(5): 401-408.

Turano, C., S. Coppari, et al. (2002). "Proteins of the PDI family: unpredicted non-ER locations and functions." Journal of cellular physiology 193(2): 154-163.

Turnquist, H. R., J. L. Petersen, et al. (2004). "The Ig-like domain of tapasin influences intermolecular interactions." Journal of immunology 172(5): 2976-2984.

Urade, R. and M. Kito (1992). "Inhibition by acidic phospholipids of protein degradation by ER-60 protease, a novel cysteine protease, of endoplasmic reticulum." FEBS letters 312(1): 83-86.

Urade, R., M. Nasu, et al. (1992). "Protein degradation by the phosphoinositide-specific phospholipase C-alpha family from rat liver endoplasmic reticulum." The Journal of biological chemistry 267(21): 15152-15159.

Urade, R., T. Oda, et al. (1997). "Functions of characteristic Cys-Gly-His-Cys (CGHC) and Gln-Glu-Asp-Leu (QEDL) motifs of microsomal ER-60 protease." Journal of biochemistry 122(4): 834-842.

Urade, R., H. Okudo, et al. (2004). "ER-60 domains responsible for interaction with calnexin and calreticulin." Biochemistry 43(27): 8858-8868.

van Endert, P. M., R. Tampe, et al. (1994). "A sequential model for peptide binding and transport by the transporters associated with antigen processing." Immunity 1(6): 491-500.

Wada, I., S. Imai, et al. (1995). "Chaperone function of calreticulin when expressed in the endoplasmic reticulum as the membrane-anchored and soluble forms." The Journal of biological chemistry 270(35): 20298-20304.

Ware, F. E., A. Vassilakos, et al. (1995). "The molecular chaperone calnexin binds Glc1Man9GlcNAc2 oligosaccharide as an initial step in recognizing unfolded glycoproteins." The Journal of biological chemistry 270(9): 4697-4704. 
Wearsch, P. A. and P. Cresswell (2007). "Selective loading of high-affinity peptides onto major histocompatibility complex class I molecules by the tapasin-ERp57 heterodimer." Nature immunology 8(8): 873-881.

Wearsch, P. A., D. R. Peaper, et al. (2011). "Essential glycan-dependent interactions optimize MHC class I peptide loading." Proceedings of the National Academy of Sciences of the United States of America 108(12): 4950-4955.

Williams, A. M. and C. A. Enns (1991). "A mutated transferrin receptor lacking asparaginelinked glycosylation sites shows reduced functionality and an association with binding immunoglobulin protein." The Journal of biological chemistry 266(26): 1764817654.

Williams, A. P., C. A. Peh, et al. (2002). "Optimization of the MHC class I peptide cargo is dependent on tapasin." Immunity 16(4): 509-520.

Yan, J., V. V. Parekh, et al. (2006). "In vivo role of ER-associated peptidase activity in tailoring peptides for presentation by MHC class Ia and class Ib molecules." The Journal of experimental medicine 203(3): 647-659.

Yewdell, J., C. Lapham, et al. (1994). "MHC-encoded proteasome subunits LMP2 and LMP7 are not required for efficient antigen presentation." Journal of immunology 152(3): 1163-1170.

Yewdell, J. W., L. C. Anton, et al. (1996). "Defective ribosomal products (DRiPs): a major source of antigenic peptides for MHC class I molecules?" Journal of immunology 157(5): 1823-1826.

York, I. A., S. C. Chang, et al. (2002). "The ER aminopeptidase ERAP1 enhances or limits antigen presentation by trimming epitopes to 8-9 residues." Nature immunology 3(12): 1177-1184.

Zarling, A. L., C. J. Luckey, et al. (2003). "Tapasin is a facilitator, not an editor, of class I MHC peptide binding." Journal of immunology 171(10): 5287-5295.

Zhang, Q. and R. D. Salter (1998). "Distinct patterns of folding and interactions with calnexin and calreticulin in human class I MHC proteins with altered N-glycosylation." Journal of immunology 160(2): 831-837.

Zhang, W., P. A. Wearsch, et al. (2011). "A role for UDP-glucose glycoprotein glucosyltransferase in expression and quality control of MHC class I molecules." Proceedings of the National Academy of Sciences of the United States of America 108(12): 4956-4961.

Zhang, Y., G. Kozlov, et al. (2009). "ERp57 does not require interactions with calnexin and calreticulin to promote assembly of class I histocompatibility molecules, and it enhances peptide loading independently of its redox activity." The Journal of biological chemistry 284(15): 10160-10173. 


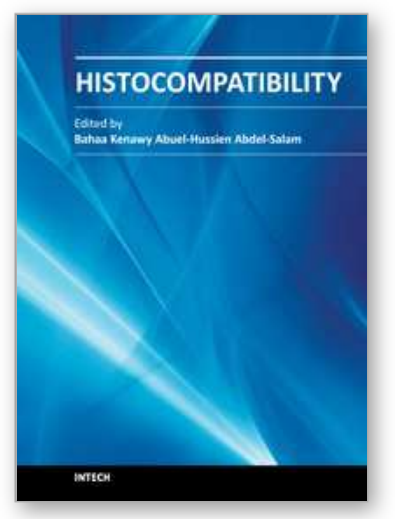

\author{
Histocompatibility \\ Edited by Dr. Bahaa Abdel-Salam
}

ISBN 978-953-51-0589-3

Hard cover, 188 pages

Publisher InTech

Published online 02, May, 2012

Published in print edition May, 2012

This book presents some recent researches related to histocompatibility for scientists interested in this field. It includes 10 chapters, in different topics, prepared by Sundararajulu Panneerchelvam and Mohd Nor Norazmi; Giada Amodio and Silvia Gregori; Adema Ribic; Bahaa K. A. Abdel-Salam; Kai-Fu Tang; Roberto Biassoni, Irene Vanni and Elisabetta Ugolotti; Wei-Cheng Yang, Lien-Siang Chou and Jer-Ming Hu; Shatrah Othman and Rohana Yusof; Masahiro Hirayama, Eiichi Azuma and Yoshihiro Komada; Gustav Roder, Linda Geironson, Elna Follin, Camilla Thuring and Kajsa Paulsson.

\title{
How to reference
}

In order to correctly reference this scholarly work, feel free to copy and paste the following:

Martha E. Mora-Herrera, Humberto Lopez-Delgado, Ernestina Valadez-Moctezuma and lan M. Scott, Gustav Roder, Linda Geironson, Elna Follin, Camilla Thuring and Kajsa Paulsson (2012). MHC Class I Quality Control, Histocompatibility, Dr. Bahaa Abdel-Salam (Ed.), ISBN: 978-953-51-0589-3, InTech, Available from: http://www.intechopen.com/books/histocompatibility/mhc-class-i-quality-control

\section{INTECH}

open science | open minds

\section{InTech Europe}

University Campus STeP Ri

Slavka Krautzeka 83/A

51000 Rijeka, Croatia

Phone: +385 (51) 770447

Fax: +385 (51) 686166

www.intechopen.com

\section{InTech China}

Unit 405, Office Block, Hotel Equatorial Shanghai

No.65, Yan An Road (West), Shanghai, 200040, China

中国上海市延安西路65号上海国际贵都大饭店办公楼405单元

Phone: +86-21-62489820

Fax: +86-21-62489821 
(C) 2012 The Author(s). Licensee IntechOpen. This is an open access article distributed under the terms of the Creative Commons Attribution 3.0 License, which permits unrestricted use, distribution, and reproduction in any medium, provided the original work is properly cited. 